\title{
Fault Detection and Isolation of Automotive Air Conditioning Systems using First Principle Models
}

\author{
Quansheng Zhang*, Marcello Canova \\ Center for Automotive Research, \\ The Ohio State University \\ 930 Kinnear Road, \\ Columbus, OH, 43212, United States
}

\begin{abstract}
Although model-based Fault Detection and Isolation (FDI) has become a common design tool in automotive fields, its application to automotive Air Conditioning $(\mathrm{A} / \mathrm{C})$ systems based upon vapor compression cycles is limited due to the lack of control-oriented models characterizing the refrigerant phase change. The emergence of Moving Boundary Method (MBM) illuminates a promising way of assisting FDI scheme development, because common faults in automotive A/C systems, such as compressor fault, pressure transducer fault and fouling fault, can be easily incorporated by the control-oriented model developed. Out of various observed-based FDI methods, the $H_{\infty}$ filter technique, due to its robustness to model uncertainties and external disturbances, is chosen for designing FDI scheme over actuator/sensor/parameter faults. The model and the filter are connected closed-loop by an $H_{\infty}$ controller gain-scheduled to meet different cooling loads. From the closed-loop analysis results, the $H_{\infty}$ filter is capable of detecting and isolating actuator/sensor faults, as well as estimating parameter faults, even if external disturbances imposed on the air side of the evaporator exist.
\end{abstract}

Keywords: Air Conditioning System, H Infinity Filter, Moving Boundary Method, Fault Detection and Isolation

\footnotetext{
* Corresponding Author

Email address: zhang.777@osu.edu (Quansheng Zhang)
}

Preprint submitted to Control Engineering Practice

June 13, 2015 


\section{Introduction}

Timely detection and isolation of underlying faults is crucial for meeting stringent safety requirements in industry. Compared to hardware redundancy technique, model-based FDI method leads to significant cost savings. Various approaches have been applied to the residual generation problem, e.g. parity equation methods, observer-based methods or frequency domain methods, [1-4]. In observer-based FDI methods [5, 6], actuator commands and sensor measurements are exploited to design filters for generating residuals that are compared to thresholds. In particular, $H_{\infty}$ filters attract more interests due to their ro-

bustness to model uncertainties and external disturbances. An optimization problem is formulated to minimize the influences of measurement noises, external disturbances and model uncertainties on the residuals and to maximize the effects of the faults on the residuals. A standard framework for the problem formulation and solution was proposed in $[7,8]$, with applications to aircraft longitudinal motion $[9,10]$.

Model-based FDI method has widespread applications in automotive engines, especially air path systems and fuel path systems $[11,12]$. In contrast, its application to auxiliary loads, such as A/C systems, has not been addressed, with limited publications available. However, Vapor Compression Cycle (VCC), the thermodynamic process obeyed by automotive A/C system, is widely applied in many industry fields, such as refrigerators, air conditioners, heat pumps and chillers. Early works on VCC fault diagnosis heavily relied on simplified models, resulting into intense calibration efforts and poor performances during transient [13]. For instance, a simplified physical model of a small heat pump system was used to generate predictions, whose differences from monitored observations were transformed into useful statistical quantities to be compared with predetermined thresholds [14]. A statistical rule-based fault detection and diagnostic method for A/C equipment was developed in [15], and demonstrated in limited testing with a roof-top air conditioner in [16] together with a fault 
evaluation method. Steady-state data representing normal operations were used to develop seven polynominal models characterizing the $\mathrm{A} / \mathrm{C}$ performance and determine the statistical thresholds for fault detection, while transient data with faults were used to evaluate fault diagnosis performance [17]. However, since they lack control-oriented models describing the thermofluid dynamics of the phase-changing refrigerant in heat exchangers, it is difficult to achieve balances between physical accuracy and computation time of the designed FDI algorithms $[18,19]$.

A lumped-parameter modeling approach named MBM method of developing control-oriented models for heat exchangers with phase change changing fluid was proposed in [20-22], where the refrigerant is lumped according its phase status, namely pure vapor, pure liquid and mixture of vapor and liquid. Differential equations describing the mass and energy balances of the phase change process were developed. The MBM A/C model offers the advantage of capturing the transient behavior of the system, and reducing the simulation time without sacrificing physical accuracy.

In this paper, a control-oriented model derived from first principles is used to design an FDI scheme on an automotive A/C system. A brief literature review is given in Section II. Exemplary actuator/sensor/parameter faults are modeled and merged into the MBM A/C model in Section III. The closed-loop system composed of the control-oriented model, the output-tracking controller and the FDI filter is presented in Section IV. The closed-loop performances are evaluated over model uncertainties and external disturbances in Section V, with both abrupt and incipient faults considered. A conclusion is given in Section VI.

\section{Recent Development of VCC Fault Diagnosis}

According to a comprehensive literature in [23, 24], FDI method can be generally classified into two approaches, namely data-driven approaches and modelbased approaches (quantitative or qualitative). Data-driven approaches need a 
large amount of training data representing both normal and faulty operation, as well as a thorough understanding of the system and expertise in statistics. The resulted models are specific to the system for which they are trained and cannot be extrapolate beyond the range of the tracing data. In contrast, strengths of FDI schemes based on quantitative models allows to model both normal and faulty operation based on first principles, such that the transient behavior of the systems is captured more precisely than any other modeling technique. In other words, they are particularly important for capturing faults during transient operation. Model-based approaches ranges from empirical models, simplified models and physics-based models. Following the above classification criterion, recent developments of FDI schemes on VCC applications during last decade are presented in the order of model complexity.

Empirical models are simplest, even sometimes static. In $[25,26]$, a gray box modeling approach is adopted to capture the influence of both operating conditions and faults on system performance. The steady-state heat exchangers are divided into regions according to the refrigerant phase, and each region is modeled with $\epsilon-N T U$ methods under a crossflow configuration. The static model describing the input-output relationship is useful for diagnosing multiplesimultaneous faults in VCC equipment with decoupling feature exploited and virtual sensor developed [27].

Simplified models neglect some dynamics under certain assumptions. A four-state nonlinear model of a supermarket refrigeration system is built using a lumped-parameter approach in [28]. A bank of Extended Kalman Filters (EKFs) is constructed for isolating two temperature sensor faults in the types of drift, offset, freeze and hard-over, and a multi-model adaptive estimation method is employed to handle parametric fault caused by freeze-over or dirty built up in [29]. However, the isolation between sensor faults and parametric faults cannot be handled by the current scheme, and is compensated by a bank of Unknown Input Observers (UIOs) constructed in [30], where one state variable is treated as a system unknown input. Unfortunately, the control-oriented model introduced [28] oversimplified the heat transfer process between the refrigerant 
and the air, as the refrigerant loop excluding the evaporator is not modeled and assumed as known boundary conditions.

The dynamic response of a chiller to the change of working conditions is captured using a lumped-parameter model is built in [31], in which four first-order differential equations are built to represent the dynamics of thermal storages at the inlet and outlet of the condenser and evaporator, respectively. A new semiphysical subcooling model is adopted to represent the condensing region and subcooling region in the heat exchanger [32]. By analyzing the changing trends of two proposed performance indexes, namely the normalized heat transfer coefficient and the fictitious subcooling temperature, the pattern in fault conditions can be obtained. Similarly, a strategy, in which six physical performance indices are used to describe the health conditions and thus indicate chiller faults, is validated against field data from a centrifugal chiller in a real building [33]. Although the model presents the effects of working conation changes on compressor load, it cannot describe the dynamic performance of chiller operation.

Physics-based models have been recently used for analyzing fault effects. In [34], the possibility of using more complex moving-boundary models for FDI in subcritical VCC equipment is explored. A linearized form of the model is used to explore the sensitivity of each output to fault conditions of evaporator frosting, refrigerant and valve leakages; however, no practical FDI algorithm is implemented. The static component based fault detection method is also tested for a transcritical refrigeration system for faults such as over/undercharge, evaporator fouling, gas cooler fouling and compressor valve leakage [35]. The method is also applicable to large transient of the refrigerant during start-up or shut-down process [36]. However, physics-based models, as far as the authors know, have never been used for design FDI scheme during transient.

\section{Fault Modeling Using MBM A/C Model}

Commonly observed faults are labelled at corresponding locations in the A/C loop and incorporated into mathematical models describing thermofluid 
dynamics.

\subsection{Overview of $A / C$ System Faults}

A basic automotive A/C system is composed of four primary components, namely evaporator, compressor, condenser and expansion valve as illustrated in Figure 1. The vapor compression cycle removes heat from the air flowing to the cabin through the evaporator, as the refrigerant evaporates from twophase (TP) status into superheated (SH) status, and rejects heat to the air flowing through the condenser, as the refrigerant condenses from superheated (SH) status into subcooled (SC) status through two-phase (TP) status. The enthalpy, mass flow rate and pressure, are exchanged by the four components. Basically, the two heat exchangers set the pressures of the system, while the compressor and expansion valve determine the mass flow rates at the inlet and outlet of the evaporator and condenser.

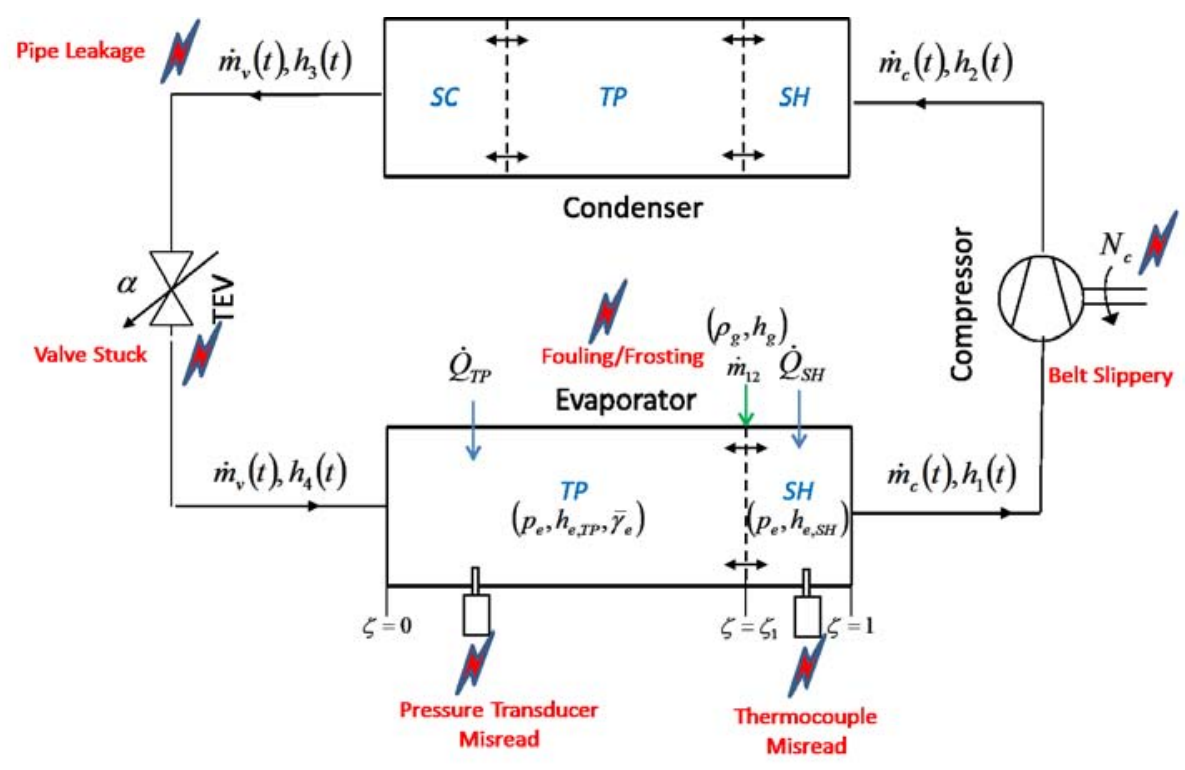

Figure 1: A/C System Components and Possible Faults.

Typical faults occurring in vapor compression systems summarized in [13] are labeled in Figure 1. For instance, compressor faults can be caused by motor 
winding failure and unbalanced voltage, or by refrigerant contamination and liquid slugging. Stepper motor and electronic controller failures could make the expansion valve malfunction. Fouling and frosting are major concerns on the surface of the heat exchangers. Refrigeration leak may occur in the pipeline. In addition to system faults, the pressure transducers and thermocouples for experimental measurement are also possible fault sources. According to the terminology of the model-based FDI, the above faults are classified into three categories, namely actuator faults (e.g. compressor fault and valve fault), sensor faults (e.g. pressure transducer fault and thermocouple fault) and parametric faults (e.g. pipe leakage and fouling). From a fault observability standpoint, the number of simultaneously detectable faults is determined by the total number of sensors available. Due to hardware limitations, the faults of interest include one actuator fault (compressor fault), sensor fault (pressure transducer fault), as well as one parametric fault (evaporator fouling fault).

An in-depth analysis of the influences of the faults on the automotive A/C system performance can be done with a control-oriented model. In a wellestablished modeling framework, the compressor and valve are modeled as static components. The dynamics related to the heat and mass transfer inside the heat exchangers are described using the MBM method [20, 22], describing the mass and energy conservation for transient one-dimensional flow. After derivations detailed in [37] and not included here for brevity, the final mathematical equations describing system dynamics, together with faults, are in the descriptor form,

$$
\begin{array}{r}
Z\left(x, f_{a}\right) \frac{d x}{d t}=f\left(x, f_{a}, u, v, f_{N}\right) \\
y=g\left(x, f_{a}, f_{p}\right)
\end{array}
$$

The inputs are the compressor rotation speed and expansion valve opening percentage, $u=\left[\begin{array}{ll}N_{c} & \alpha\end{array}\right]^{T}$. The boundary conditions are the variables describing the air side of the heat exchangers, and could be treated as unknown disturbances, $v=\left[\begin{array}{ll}\dot{m}_{e a} & T_{e a, i n}\end{array}\right]^{T}$. The state vector describing the evaporator status includes 5 variables as: $x_{e}=\left[\begin{array}{lllll}\zeta_{e 1} & p_{e} & h_{e 2} & T_{e 1 w} & T_{e 2 w}\end{array}\right]^{T}$. Finally, the out- 
puts are the evaporator pressure and superheat temperature, $y=\left[\begin{array}{ll}p_{e} & S H\end{array}\right]^{T}$, which are only functions of refrigerant states. The $Z$ matrix and $f$ vector are complex expressions of refrigerant properties, heat transfer coefficients and geometric parameters [37].

\subsection{Actuator Fault}

The compressor fault $f_{N}$ belongs to actuator faults. In compressor, the mass flow rate $\dot{m}_{c}$ and outlet enthalpy $h_{2}$ are defined respectively as:

$$
\dot{m}_{c}=\eta_{v} V_{d} \rho_{1} \omega_{c}, \quad h_{2}=\frac{h_{2 s}-h_{1}}{\eta_{s}}+h_{1}
$$

where $V_{d}$ is the compressor displacement, $\rho_{1}, h_{1}$ the refrigerant density and enthalpy at the compressor inlet, $\omega_{c}$ the compressor speed in $\mathrm{rad} / \mathrm{sec}$ and $h_{2 s}-$ $h_{1}$ is the isentropic enthalpy difference. Thus, the mass flow rate and enthalpy change are mainly determined by the compressor rotation speed $\omega_{c}$ in $\mathrm{rad} / \mathrm{s}$ or $N_{c}$ in rpm. If the compressor fault $f_{N}$ is modeled as an additive fault, the actual compressor speed $N_{A C}$ is different from the commanded compressor speed $N_{c m d}$ from the controller, $N_{A C}=N_{c m d}+f_{N}$. Note that although $f_{N}$ is additive, it acts nonlinearly due to its influence on $\eta_{v}$ and $\eta_{s}$.

\subsection{Sensor Fault}

Two sensors are installed into the evaporator for control purposes, namely one pressure transducer and one thermocouple. The thermocouple is installed at the outlet of the evaporator in order to measure the degree of superheated temperature. Obviously, both faults are closely related to the refrigerant dynamics involving of pressure and temperature. Hence, the mass balance equation and energy balance equation for the refrigerant in the superheated region of the evaporator are presented:

$$
\begin{aligned}
& -\left(\frac{\rho_{e, S H}-\rho_{g}}{\rho_{e, S H}}\right) \frac{d \zeta_{1}}{d t}+\frac{1}{\rho_{e, S H}} \frac{\partial \rho_{e, S H}}{\partial p_{e}} \frac{d p_{e}}{d t} \cdot\left(1-\zeta_{1}\right)+\frac{1}{\rho_{e, S H}} \frac{\partial \rho_{e, S H}}{\partial h_{e, S H}} \frac{d h_{e, S H}}{d t} \cdot\left(1-\zeta_{1}\right) \\
& =\frac{\dot{m}_{12}}{\rho_{e, S H} V_{e}}-\frac{\dot{m}_{c}}{\rho_{e, S H} V_{e}}
\end{aligned}
$$




$$
\begin{aligned}
& -\frac{\rho_{g}\left(h_{g}-h_{e, S H}\right)}{\rho_{e, S H}} \frac{d \zeta_{1}}{d t}+\frac{1}{\rho_{e, T P}} \frac{d p_{e}}{d t} \cdot\left(1-\zeta_{1}\right)-\frac{d h_{e, S H}}{d t} \cdot\left(1-\zeta_{1}\right) \\
& =\frac{\dot{m}_{12}}{\rho_{e, S H} V_{e}}\left(h_{g}-h_{e, S H}\right)-\frac{\dot{m}_{c}}{\rho_{e, S H} V_{e}}\left(h_{1}-h_{e, S H}\right)+\frac{\dot{Q}_{S H}}{\rho_{e, S H} V_{e}}
\end{aligned}
$$

where $p_{e}$ is the evaporator pressure, $\zeta_{1}$ the two-phase region normalized tube length, $h_{e, S H}$ the enthalpy of the refrigerant at the tube exit, $\dot{m}$ mass flow rate, $\dot{Q}$ heat transfer rate, $\rho$ density. All these variables have been labeled in Figure 1.

A high-level understanding these differential equation is possible without diving into the mathematical details. The Left Hand Side (LHS) represents the variation of independent states of the refrigerant, $\zeta_{1}, p_{e}, h_{e, S H}$, and Right Hand Side (RHS) the exchanger of mass and enthlpy at the inlet and outlet of individual phase region, $\dot{m}, h$, as well as the heat transfer along the wall of corresponding region $\dot{Q}$. The terms multiplying the state variations depend on the refrigerant inherent thermodynamic properties, hence are state-dependent. The mass and energy balances for the subcooled, two-phase and superheated region of the condenser are not shown here because of their indirect relationships to faults. The pressure transducer measurement is directly linked to one state of the differential equations, $p_{m}=p_{e}$ if no fault exists, or $p_{m}=p_{e}+f_{p}$ if a fault emerges.

\subsection{Parameter Fault}

The fouling on the tube exterior surface reduces heat transfer rates as a result of reduced heat transfer coefficient. The fouling dynamics is closely related to the wall temperature dynamics of the heat exchangers. A lumped thermal mass approach is adopted to describe the temperature of the walls separating the refrigerant control volume from the external air flow:

$$
\rho_{w} A_{w} c_{w}\left[L \frac{d T_{w}}{d t}+T_{w}\left(v_{b 2}-v_{b 1}\right)\right]+\rho_{w} A_{w} c_{w}\left(T_{w 1} v_{b 1}-T_{w 2} v_{b 2}\right)=\dot{Q}_{\text {in }}-\dot{Q}_{o u t}
$$

where $A_{w}$ is a representative cross-sectional area. The internal and external heat transfer models assume pure convection occurring between the wall and 
the fluids in contact. In case of the external heat transfer (air to wall), the following equation is used:

$$
\dot{Q}_{\text {out }}=\alpha_{\text {out }}\left(\pi D_{\text {out }} L\right)\left(T_{a}-T_{w}\right)
$$

where $D_{\text {out }}$ is the hydraulic diameter. The fouling fault is modeled as $\alpha_{\text {out }, a c t}=$ $\alpha_{\text {out }}+f_{\alpha}$, where $\alpha_{\text {out }}$ is a theoretical value computed using heat transfer correlations for compact heat exchangers, and $\alpha_{\text {out,act }}$ is the actual heat transfer coefficient of the heat exchanger.

\section{Experiment System}

Provided that commands to compressor speed $N_{c}$ and valve position $\alpha$, as well as measurements of evaporator pressure $p_{e}$ and superheat temperature $S H$ are available for the control module and the FDI module, as depicted in Figure 2 , the instrumented test vehicle enables accomplishing tasks of

1. modeling and calibrating a MBM A/C model [37, 38];

2. designing an $H_{\infty}$ controller for output tracking [37, 38];

3. developing FDI schemes in the closed-loop of plant, controller and filter.

When the MBM A/C model is validated, the air mass flow rates and temperatures on the evaporator side $\dot{m}_{e a}, T_{e o}$ and condenser side $\dot{m}_{c a}, T_{c o}$, are either controllable or measurable. The output signals, such as air temperatures leaving the heat exchangers $T_{e o}, T_{c o}$ and tube wall temperatures $T_{e w}, T_{c s}$ are predicable using the MBM A/C model. Since the A/C system dynamics change significantly over working points, the $H_{\infty}$ controller dynamics is supposed to be scheduled accordingly. Both the model validation and control design have been accomplished in [37], and the main results are summarized for the convenience of the $H_{\infty}$ filter development.

An automotive A/C system in a Minivan is studied. The test vehicle was instrumented with an ETAS ES1000 system interfaced with the Engine Control Unit (ECU) to allow for acquisition of engine torque and speed, and to control 


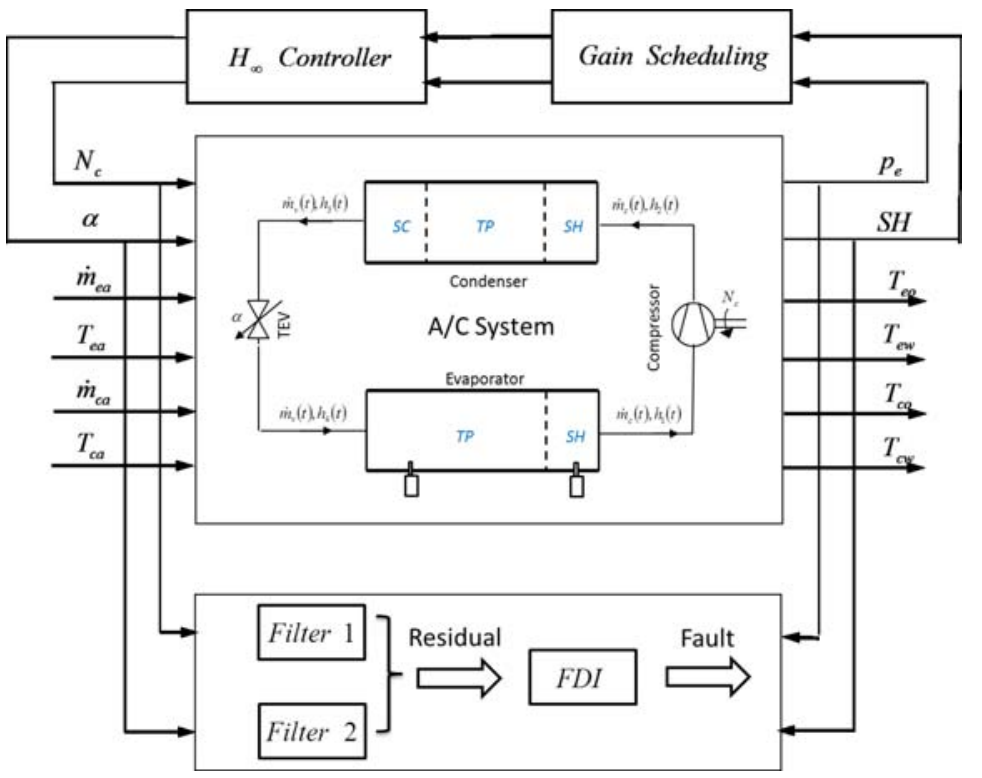

Figure 2: Interconnections of Plant, Controller and FDI.

the radiator fan and cabin blower. The vehicle was installed on a chassis dynamometer, allowing for the characterization of the system both at steady-state conditions and during driving cycles.

Leveraging upon the available experimental setup, the model was calibrated using the data collected during the tests when vehicle/engine speeds are maintained at nominal steady state, and verified with reference to the SC03 Air Conditioning Cycle whose vehicle speed trace for this regulatory driving cycle. After validation, the nonlinear $\mathrm{A} / \mathrm{C}$ model is linearized at three operating conditions, corresponding to low, medium and high cooling loads. The system matrices of the LTI A/C model are provided in [37, 38].

The robust $H_{\infty}$ controller is designed to track prescribed trajectories of two output variables, namely the evaporator pressure $p_{e}$, and the superheat temperature $S H$. Meanwhile, the controller should reject disturbances caused by air mass flow rate at the evaporator, $\dot{m}_{e a}$. The system matrices of the final $H_{\infty}$ controller after model order reduction, together with its derivation process, are given in $[37,38]$. 
The $H_{\infty}$ fault diagnosis problem is to find a stable filter $F$ such that 1) the performance error is minimized under all possible uncertainties $\Delta ; 2$ ) the faults effect on the residuals is maximized with disturbance effect minimized. The second objective is equivalent to minimization of the error between the residual and the weighted fault. Hence, a standard performance index can be given as follows:

$$
\sup _{0<\|\Phi\|_{2}<\infty} \frac{\left\|r e s-T_{f_{i d}} f\right\|_{2}}{\|\Phi\|_{2}}
$$

and the matrices $T_{f_{i d}}$ and $\Phi$ vary depending on the type of problems. Please refer to $[7,8]$ for theoretical backgrounds and $[9,10]$ for application examples.

\section{Results and Analysis}

Following the general FDI schemes introduced, two separated $H_{\infty}$ filters, namely an actuator/sensor FDI filter and a parameter FDI filter, will be designed to detect the compressor fault and the pressure transducer fault, as well as to estimate the fouling fault using the MBM A/C model.

\subsection{Actuator and Sensor FDI}

For the FDI scheme, the standard $H_{\infty}$ synthesis, which requires selecting appropriate weighting functions for optimization problem formulation, enables that the generated residuals to follow the faulty signals dynamically. The reference fault weights $W_{\text {fault,act }}, W_{\text {fault,sen }}$ and estimation error weights $W_{\text {err }, a c t}, W_{\text {err,sen }}$ are selected in order to minimize the error at low frequencies and relax the constraints at higher frequencies. The $H_{\infty}$ filter calculated based on the augmented plant model is inherently high-order. From the analysis of the singular values of system matrices of the $H_{\infty}$ filter, three states dominate the system dynamics. Hence, the $H_{\infty}$ filter after model order reduction is given 
below.

$$
\begin{aligned}
A_{F} & =\left(\begin{array}{ccc}
-6.24 e-6 & 0.004616 & -0.0006508 \\
-0.004895 & -0.08784 & 0.08659 \\
0.0008169 & 0.08861 & -0.2091
\end{array}\right) \\
B_{F} & =\left(\begin{array}{cccc}
-4.152 e-5 & -0.001717 & 0.0001151 & -0.2094 \\
-0.0004144 & -0.1061 & 0.004668 & -21.91 \\
-0.003091 & 0.2544 & -0.004444 & 12.53
\end{array}\right) \\
C_{F} & =\left(\begin{array}{ccc}
0.1383 & -21.87 & 12.43 \\
-0.5172 & -1.187 & -1.66
\end{array}\right)
\end{aligned}
$$

Suitable thresholds are selected to allow for categorizing the fault signatures as 0 (no fault detected) and 1 (fault detected). Table 1 summarizes the FDI scenario for the actuator/sensor faults of the A/C plant. Theoretically, the selection criterion depends on the probability density function (PDF) of faulty and non-faulty signals. Because the faulty and non-faulty PDFs may have significant overlap, it might result in a certain probability of error either from false alarm or missed detections. Thus, an optimal threshold must be determined using hypothesis testing, such that the probability of error, as an integral of the overlapped region of the PDF, is minimized [39]. Given a nominal PDF and a maximum allowable probability of error, e.g. 5\%, the minimum level of detectable fault can be calculated, e.g. $10 \mathrm{rpm}$ for compressor fault and $1 \mathrm{kPa}$ for pressure transducer fault. In order to reduce the complexity of thresholds selection, the differentiation between faulty and non-faulty residuals is enlarged intentionally by injecting more significant fault, e.g. $50 \mathrm{rpm}$ for compressor fault and $10 \mathrm{kPa}$ for pressure transducer fault.

\begin{tabular}{|c|c|c|}
\hline & res $_{1}$ & res $_{2}$ \\
\hline No Fault & 0 & 0 \\
\hline Sensor Fault & 0 & 1 \\
\hline Actuator Fault & 1 & 0 \\
\hline Both Faults & 1 & 1 \\
\hline
\end{tabular}

Table 1: Fault Signatures for A/C System. 


\subsubsection{Performance Evaluation}

A test of the performance of the $H_{\infty}$ filter designed is shown in Fig. 3, with the actual sensor measurements $\left(p_{e}, S H\right)$, commands to actuators $\left(N_{c}, \alpha\right)$, and corresponding residuals $\left(r e s_{1}, r e s_{2}\right)$ recorded. The robustness of the filter ensures that the residual generation process becomes less sensitive to some discrepancies between the actual A/C plant and the LTI model used during the filter design process. Hence, the working point is taken away from the design point corresponding to the medium cooling load, where inputs are set to $N_{c}=1000 \mathrm{rpm}$ and $\alpha=40 \%$, and corresponding outputs are regulated to $p_{e}=250 \mathrm{kPa}$ and $S H=20{ }^{\circ} \mathrm{C}$. The A/C system settles down before $T=400$ sec, resulting an increased evaporator pressure by $5 \mathrm{kpa}$ and a reduced superheat temperature by $2.5^{\circ} \mathrm{C}$.

The actuator fault is a fixed value of positive $50 \mathrm{rpm}$ in the compressor speed $N_{c}$ injected at $T=400 \mathrm{~s}$ and removed at $T=500 \mathrm{~s}$. As seen from Fig. 3 , the actuator fault causes the first residual $r s_{1}$ to cross the threshold, while the second residual $\mathrm{res}_{2}$ changes negligibly. Because the $H_{\infty}$ controller is inherently a passive fault tolerant controller, the commanded compressor speed $N_{c}$ sent to the actuator is reduced by around $50 \mathrm{rpm}$ in order to compensate the actuator fault. The sensor fault is a fixed value of positive $10 \mathrm{kPa}$ in the pressure transducer $p_{e}$ injected at $T=700 \mathrm{~s}$ and removed at $T=800 \mathrm{~s}$. As seen from Fig. 3, the sensor fault causes the second residual res $_{2}$ to cross the threshold, while the first residual res $_{1}$ changes negligibly. Although the measured evaporator pressure jumps by $10 \mathrm{kPa}$ abruptly, the robustness of the $H_{\infty}$ controller forces the A/C plant to move to another working point by modulating the two actuators, in order to counteract the influence of the pressure transducer misreading and guarantee that it returns to $p_{e}=255 \mathrm{kPa}$.

Besides the scenario with an abrupt change of an actuator/sensor fault, the $H_{\infty}$ filter is also capable of estimating the faulty actuator/sensor facing an incipient change. As before, the working point is moved from the one corresponding to the medium cooling load for the first $400 \mathrm{sec}$. A gradual deteriorated actua- 

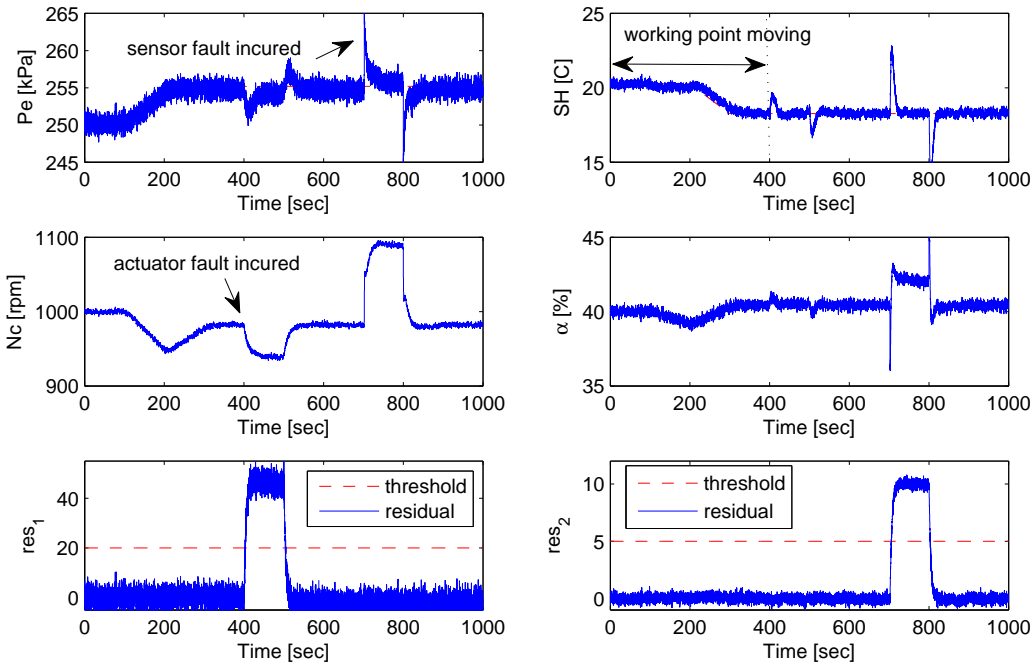

Figure 3: Plant Outputs, Inputs and Filter Residuals for Abrupt Fault .

tor, starting at healthy status at $400 \mathrm{sec}$ and ending at 5\% faulty signal at 1000 sec, is injected to represent an incipient actuator fault. Similarly, a gradual deteriorated sensor, starting at healthy status at $700 \mathrm{sec}$ and ending at $4 \%$ faulty signal at $1000 \mathrm{sec}$, is injected to represent an incipient sensor fault. From figure 4, the first residual $r e s_{1}$ estimates the actuator fault, and the second residual $r s_{2}$ estimates the sensor fault very accurately. However, for the fixed thresholds specified, the $H_{\infty}$ filter cannot detect the actuator fault with a delay of 200 sec, and the sensor fault with a delay of $100 \mathrm{sec}$. For instant fault detection, an $H_{\infty}$ filter with an adaptive threshold is expected.

\subsubsection{Limitations}

The designed $H_{\infty}$ filter suffers several limitations, from the forthcoming observations that the residuals generated are sensitive to boundary conditions and plant variations. In Figure 5, the air velocity through the evaporator deviates from the nominal value $4 \mathrm{~m} / \mathrm{sec}$ sinusoidally after $400 \mathrm{sec}$, while both the actuator and sensor faults are injected as constant values at the beginning of the test. 

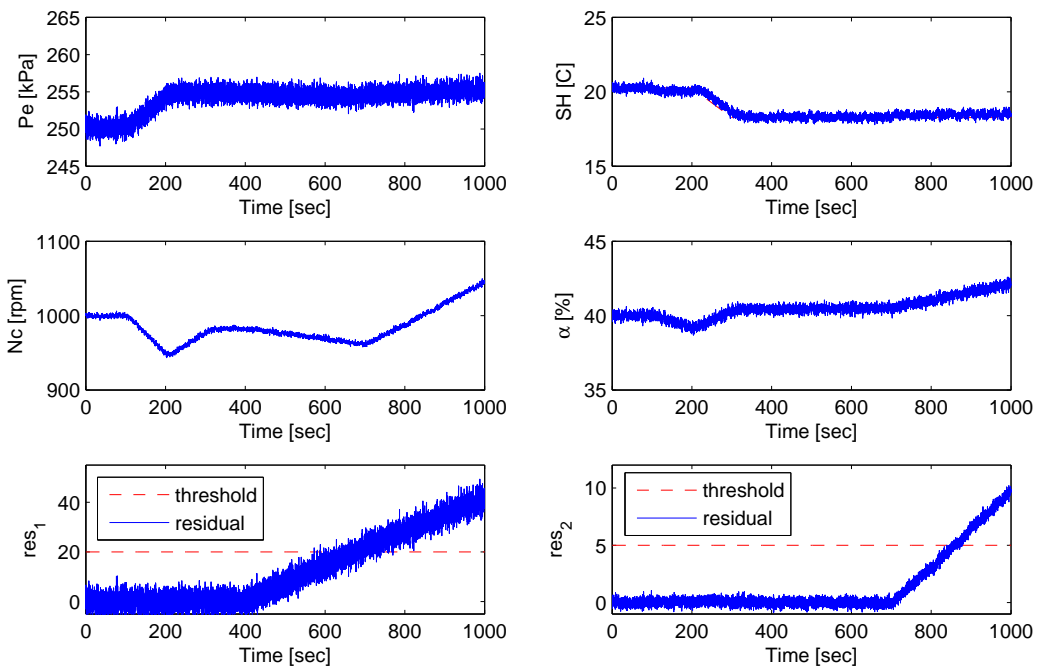

Figure 4: Plant Outputs, Inputs and Filter Residuals for Incipient Fault.

As expected, the residuals representing fault occurrences exceed the thresholds when the air velocity is constant for the first phase $(t<400 \mathrm{sec})$. For the second phase $(t>400 \mathrm{sec})$, however, as the air velocity oscillates, the residuals fall below thresholds despite that faults exist all the time, indicating that missed detection happens.

The influences of external disturbances on the residuals may be removed once additional thermcouples are installed on exterior surfaces of the heat exchangers in order to provide the wall temperatures at individual phase regions. Note that the choice of wall temperature signal is targeted for the specific disturbance coming from the air side, as the latter is independently observable from the former. Thus the control-oriented A/C system model for the $H_{\infty}$ filter design has more outputs available than before, allowing that the synthesis process has additional authority of rejecting external disturbances. After the new filter replaces the original one, the residuals for both actuator fault and sensor fault change in a similar manner to those in Figure 3, while extra actuator efforts are required to counteract the effects of external disturbances. 

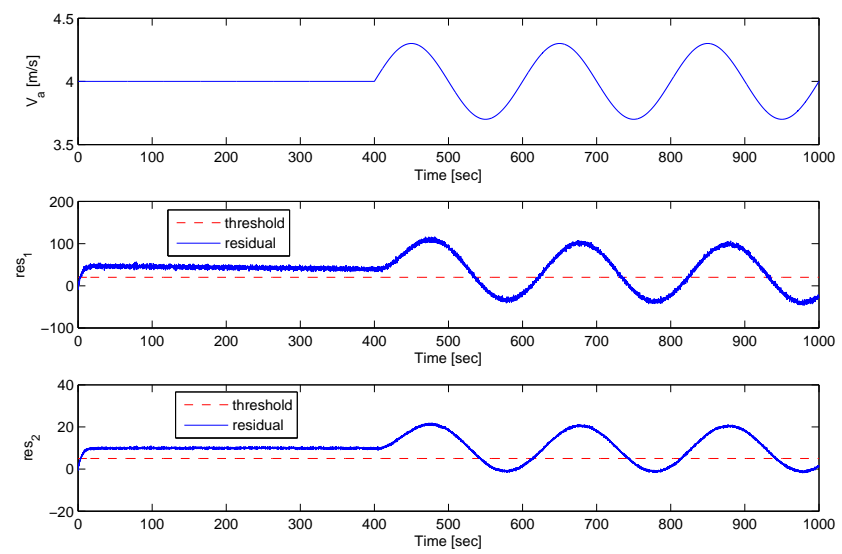

Figure 5: Influence of External Disturbance on Residual Generation.

Plant variations also affect the filter performance, even if the $H_{\infty}$ controller is gain scheduled to the LTI A/C plants obtained at different linearization points. For instance, the working point corresponding to the low cooling load is selected, and the the $H_{\infty}$ filter is designed using the same weighing functions used for the medium cooling load scenario for consistency. As before, the working point is taken away from the design point, and actuator fault and sensor fault are injected sequentially in Figure 6. From the two figures in the third row, the two residuals generated are very noisy, such that false alarms and missing alarms happen frequently with respect to fixed thresholds, especially for the residual of sensor fault $r e s_{2}$. In order to distinguish residuals from noises, additional filters modulating the gains and frequency of the original filters are added in series of the original to remove noises. From the two figures in the fourth row, two residuals after filtering are capable of detecting and isolating actuator fault injected at $400 \mathrm{sec}$ and sensor fault injected at $700 \mathrm{sec}$. In other words, the appropriate filters at low cooling load is a product of the additional filter and the original one corresponding to medium cooling load. The sensitivity of residuals to plant variables is removable if the $H_{\infty}$ filter is designed using gain-scheduling technique, such as Linear Parameter Varying (LPV) approach 
in $[40]$
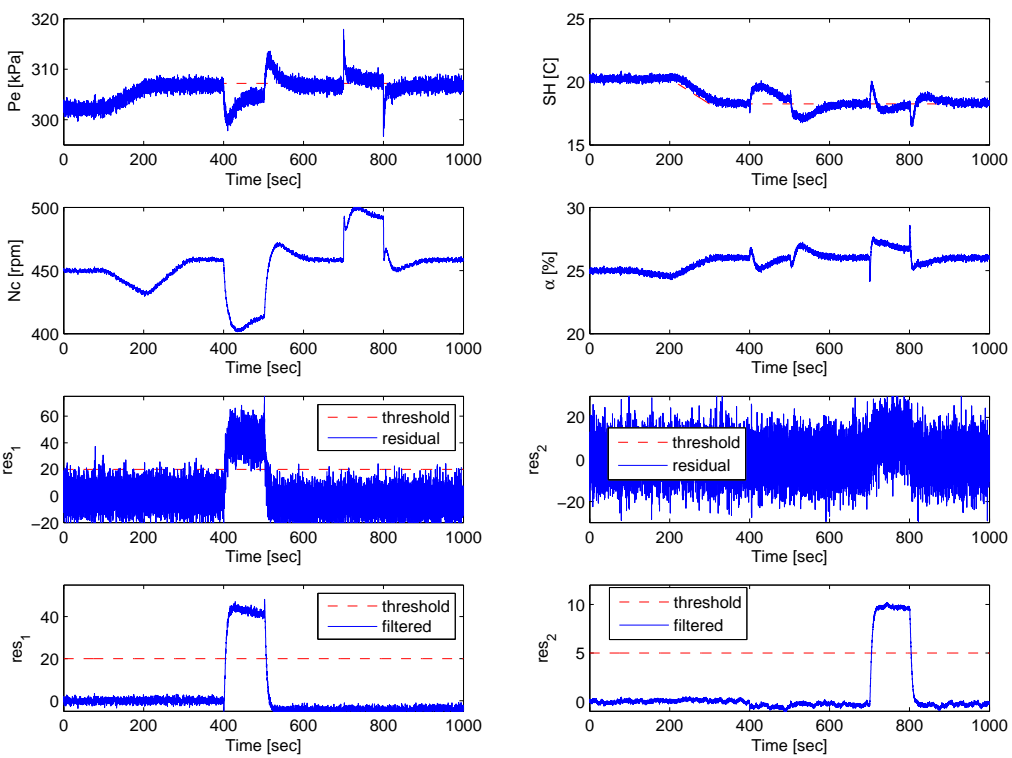

Figure 6: Plant Variation Effect on Residual Generation.

\subsection{Parametric FDI}

In the scheme of the parameter FDI scheme, the standard $\mu$ synthesis, which requires structuring the uncertainty block for optimization problem formulation, enables the generated residual has the same amplitude of the parameter fault. The uncertainty block is constructed by perturbing the nominal system around equilibrium points. After appropriate weighting functions selected, the $H_{\infty}$ filter calculated based on plant model with uncertainty block is inherently highorder. From the analysis of the singular values of the filter system matrices, three states dominate the system dynamics. Hence, the $H_{\infty}$ filter after model 
order reduction is given below.

$$
\begin{aligned}
A_{F} & =\left(\begin{array}{ccc}
-0.1535 & -0.3811 & 0.02074 \\
0.3794 & -3.514 & 0.6827 \\
0.02033 & -0.6843 & -0.03135
\end{array}\right) \\
B_{F} & =\left(\begin{array}{cccc}
0.01559 & -0.08417 & 0.005109 & -16.82 \\
-0.2407 & -1.999 & -0.02167 & 19.56 \\
-0.03282 & -0.235 & -0.003236 & 1.142
\end{array}\right) \\
C_{F} & =\left(\begin{array}{ccc}
16.82 & 19.65 & -1.162 \\
1.549 e-5 & -5.925 e-5 & 0.0001626
\end{array}\right)
\end{aligned}
$$

The above $H_{\infty}$ filter is incorporated into the closed-loop system for evaluating its performance when the corresponding parameter changes abruptly under external disturbances. As before, the working point is moved from the one corresponding to the medium cooling load for the first $400 \mathrm{sec}$. Then parameter faults, whose amplitudes are $5 \%$ and $3 \%$ of the nominal value, and time duration are $200 \mathrm{sec}$, are injected sequentially to check residual variations. Finally, a sinusoidal disturbance is added to the air velocity of the evaporator side for the ending 200 sec. The commands sent to the actuators and measurements from the sensors are recorded in Fig. 7.
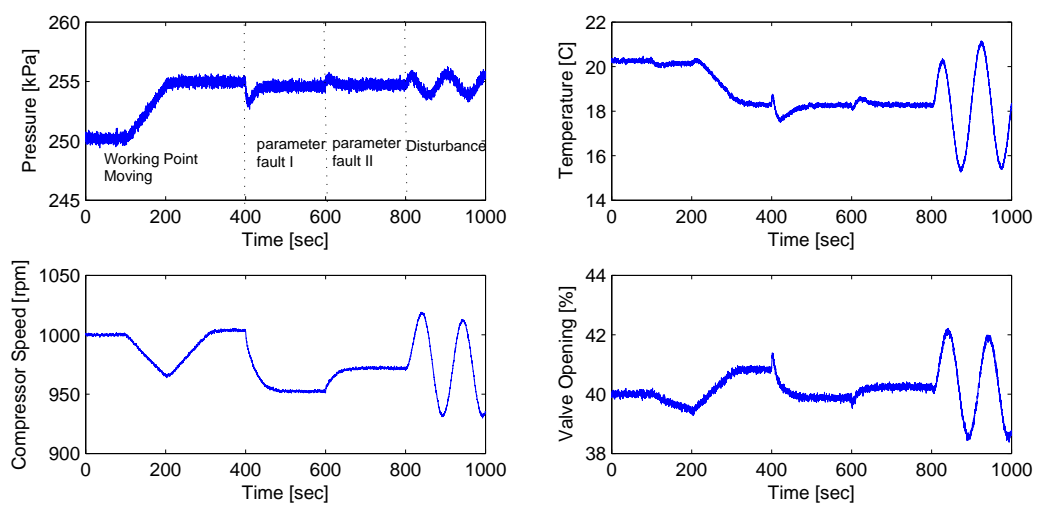

Figure 7: Plant Output and Inputs for Parameter Fault.

In Fig. 8 , the capability of the $H_{\infty}$ filter is demonstrated by showing whether 
the residual res, calculated as the ratio of $\left\|\hat{f}_{p}\right\| /\left\|\hat{z}_{p}\right\|$, follow the actual parameter variation. It is clear that the amplitudes of the changing parameter fault ( $5 \%$ and $3 \%$ sequentially) are captured by the residual signal after transient. Although external disturbances cause the estimations of $f_{p}$ and $z_{p}$ change sinusoidally, the residual representing the faulty parameter amplitude is maintained constant after $T=800 \mathrm{sec}$. Hence, the designed $H_{\infty}$ filter is capable of detecting the parameter fault under external disturbances.

incurred in the figure
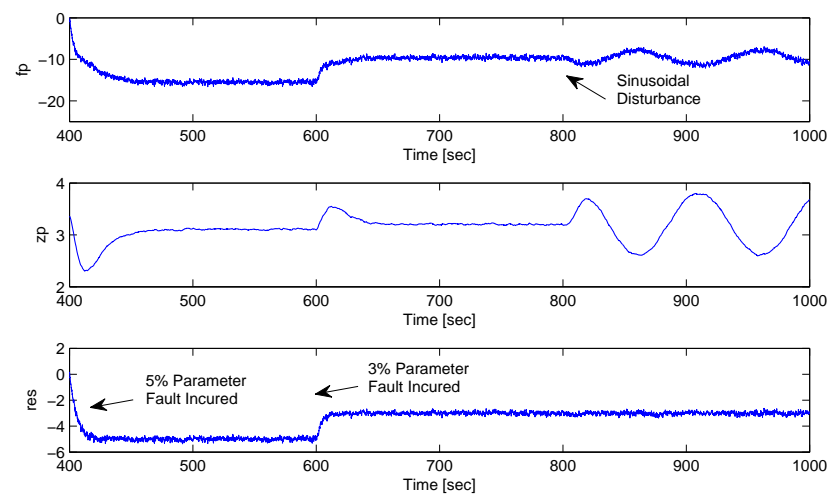

Figure 8: Residual Calculation for Abrupt Parameter Fault.

Besides the scenario with an abrupt change of a parameter fault, the $H_{\infty}$ filter is also capable of estimating the faulty parameter facing an incipient change. A gradual deteriorated parameter, starting as unfaulty status at $400 \mathrm{sec}$ and ending as $3 \%$ faulty status at $1000 \mathrm{sec}$, is injected to represent an incipient parameter fault. From Figure 9, the residual estimates the parameter fault very accurately, no matter whether the A/C plant is disturbance-free for the first $300 \mathrm{sec}$ or affected by sinusoidal fault for the second $300 \mathrm{sec}$. Therefore, it is concluded that the the $H_{\infty}$ filter designed is capable of estimating both abrupt and incipient parameter faults. 

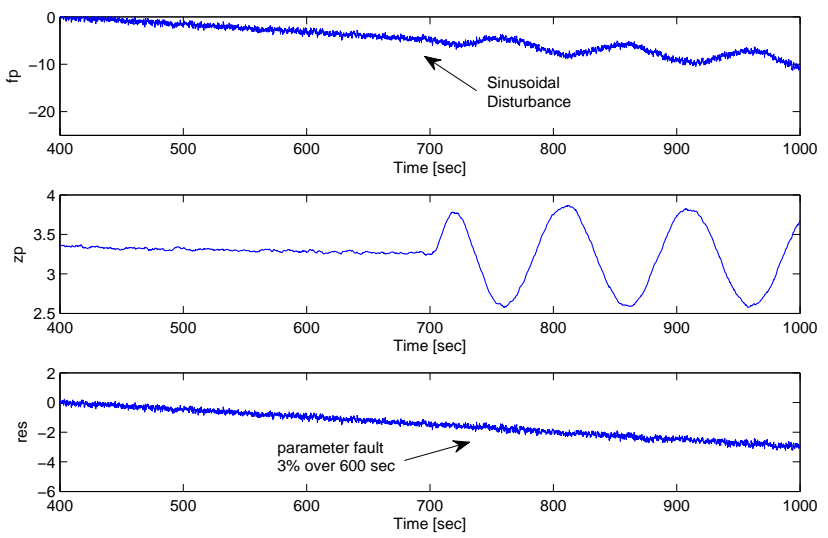

Figure 9: Residual Calculation for Incipient Parameter Fault.

\section{Conclusion}

In this paper, an $H_{\infty}$ filter based FDI method has been applied to an automotive A/C system using model-based approaches. An MBM A/C model built using first principles, for the first time, serves as a control-oriented model for distinguishing faults. The designed $H_{\infty}$ filters are capable of detecting an actuator and a sensor fault simultaneously, and estimating a parameter fault, no matter how the faults change abruptly or incipiently. The robustness of the $H_{\infty}$ filter to external disturbances is achievable by installing additional thermocouples. Besides, the sensitivity of the $H_{\infty}$ filter to plant variation might be mitigated using gain-scheduling techniques. The reason of not designing a unified $H_{\infty}$ filter based FDI scheme is that the total number of sensors available is limited. Hence, it is not capable of isolating all faults simultaneously, especially when external disturbances exist on the evaporator air side. Future work is to merge the two filters after more measurements, such as tube wall temperatures, are available. 


\section{Acknowledgment}

The work presented in this paper was funded by the U.S. Department of Energy (Award DE-EE0003347). The authors are grateful to Fiat Chrysler Automobiles (FCA) and to Dr. Timothy C. Scott for providing the information and suggestions that led to the development of this work. Furthermore, the authors gratefully acknowledge the anonymous reviewers for their insights, which contributed to strengthen the quality of this work.

\section{References}

1. Frank, P.M.. Fault diagnosis in dynamic systems using analytical and knowledgebased redundancy: A survey and some new results. Automatica 1990;26(3):459474 .

2. Isermann, R.. Model-based fault-detection and diagnosis-status and applications. Annual Reviews in control 2005;29(1):71-85.

3. Isermann, R.. Fault-diagnosis systems. Springer; 2006.

4. Ding, S.X.. Model-based fault diagnosis techniques; vol. 2013. Springer; 2008.

5. Frank, P.M., Ding, X.. Survey of robust residual generation and evaluation methods in observer-based fault detection systems. Journal of process control $1997 ; 7(6): 403-424$.

6. Patton, R., Chen, J.. Observer-based fault detection and isolation: robustness and applications. Control Engineering Practice 1997;5(5):671-682.

7. Mangoubi, R.S.. Robust estimation and failure detection: A concise treatment. Springer Publishing Company, Incorporated; 2012.

8. Stoustrup, J., H Niemann, H.. Fault estimationa standard problem approach. International Journal of Robust and Nonlinear Control 2002;12(8):649-673.

9. Marcos, A., Ganguli, S., Balas, G.J.. An application of $\mathrm{h}$ fault detection and isolation to a transport aircraft. Control Engineering Practice 2005;13(1):105119 . 
10. Marcos, A., Balas, G.J.. A robust integrated controller/diagnosis aircraft application. International Journal of Robust and Nonlinear Control 2005;15(12):531551.

11. Nyberg, M., Stutte, T.. Model based diagnosis of the air path of an automotive diesel engine. Control Engineering Practice 2004;12(5):513-525.

12. Kimmich, F., Schwarte, A., Isermann, R.. Fault detection for modern diesel engines using signal-and process model-based methods. Control Engineering Practice 2005;13(2):189-203.

13. Keir, M.C., Alleyne, A.G.. Dynamic modeling, control, and fault detection in vapor compression systems. Tech. Rep; Air Conditioning and Refrigeration Center. College of Engineering. University of Illinois at Urbana-Champaign.; 2006.

14. Wagner, J., Shoureshi, R.. Failure detection diagnostics for thermofluid systems. Journal of dynamic systems, measurement, and control 1992;114(4):699-706.

15. Rossi, T.M.. Detection, diagnosis, and evaluation of faults in vapor compression equipment 1995;.

16. Rossi, T.M., Braun, J.E.. A statistical, rule-based fault detection and diagnostic method for vapor compression air conditioners. HVACERR Research 1997;3(1):1937.

17. Breuker, M.S., Braun, J.E.. Evaluating the performance of a fault detection and diagnostic system for vapor compression equipment. HVACER Research $1998 ; 4(4): 401-425$.

18. Katipamula, S., Brambley, M.R.. Review article: methods for fault detection, diagnostics, and prognostics for building systemsa review, part i. HVACER Research 2005;11(1):3-25.

19. Katipamula, S., Brambley, M.R.. Review article: methods for fault detection, diagnostics, and prognostics for building systemsa review, part ii. HVACERR Research 2005;11(1):3-25.

20. He, X.D., Liu, S., Asada, H.H.. Modeling of vapor compression cycles for multivariable feedback control of hvac systems. Journal of dynamic systems, measurement, and control 1997;119(2):183-191. 
21. He, X., Liu, S., Asada, H., Itoh, H.. Multivariable control of vapor compression systems. HVACER Research 1998;4(3):205-230.

22. Li, B., Alleyne, A.. A dynamic model of a vapor compression cycle with shut-down and start-up operations. International Journal of refrigeration 2010;33(3):538-552.

23. Katipamula, S., Brambley, M.R.. Review article: methods for fault detection, diagnostics, and prognostics for building systemsa review, part i. HVACESR Research 2005;11(1):3-25.

24. Katipamula, S., Brambley, M.R.. Review article: Methods for fault detection, diagnostics, and prognostics for building systemsa review, part ii. HVACESR Research 2005;11(2):169-187.

25. Cheung, H., Braun, J.E.. Simulation of fault impacts for vapor compression systems by inverse modeling. part i: Component modeling and validation. HVACESR Research 2013;19(7):892-906.

26. Cheung, H., Braun, J.E.. Simulation of fault impacts for vapor compression systems by inverse modeling. part ii: System modeling and validation. HVACESR Research 2013;19(7):907-921.

27. Li, H., Braun, J.E.. Decoupling features and virtual sensors for diagnosis of faults in vapor compression air conditioners. International Journal of Refrigeration 2007;30(3):546-564.

28. Larsen, L.F., Izadi-Zamanabadi, R., Wisniewski, R.. Supermarket refrigeration system-benchmark for hybrid system control. Proc of ECC07 2007;.

29. Yang, Z., Rasmussen, K.B., Kieu, A.T., Izadi-Zamanabadi, R.. Fault detection and isolation for a supermarket refrigeration system-part one: Kalman-filterbased methods. In: The 18th World Congress of the International Federation of Automatic Control (IFAC 2011). 2011:13233-13238.

30. Yang, Z., Rasmussen, K., Kieu, A., Izadi-Zamanabadi, R.. Fault detection and isolation for a supermarket refrigeration systempart two: Unknown-inputobserver method and its extension. In: 18th IFAC World Congress. 2011:. 
31. Wang, S.w., Wang, J., Burnett, J.. Mechanistic model of centrifugal chillers for hvac system dynamics simulation. Building services engineering research and technology 2000;21(2):73-83.

32. Zhao, Y., Wang, S., Xiao, F., Ma, Z.. A simplified physical model-based fault detection and diagnosis strategy and its customized tool for centrifugal chillers. HVACER Research 2013;19(3):283-294.

33. Wang, S., Cui, J.. A robust fault detection and diagnosis strategy for centrifugal chillers. HVACER Research 2006;12(3):407-428.

34. Keir, M.C., Alleyne, A.G.. Dynamic modeling, control, and fault detection in vapor compression systems. Tech. Rep.; Air Conditioning and Refrigeration Center. College of Engineering. University of Illinois at Urbana-Champaign.; 2006.

35. Janecke, A.K.. A comparison of fault detection methods for a transcritical refrigeration system. Ph.D. thesis; Texas A\&M University; 2011.

36. Ayyagari, B.. Simulation and validation of vapor compression system faults and start-up/shut-down transients. Ph.D. thesis; Texas A\&M University; 2011.

37. Zhang, Q., Canova, M.. Modeling and output feedback control of automotive air conditioning systems. Submitted to International Journal of Refrigeration 2014;.

38. Zhang, Q., Fiorentini, L., Canova, M.. H robust control of an automotive air conditioning system. In: American Control Conference (ACC), 2014. IEEE; 2014:5675-5680.

39. Marcicki, J., Onori, S., Rizzoni, G.. Nonlinear fault detection and isolation for a lithium-ion battery management system. In: ASME 2010 Dynamic Systems and Control Conference. American Society of Mechanical Engineers; 2010:607-614.

40. Szászi, I., Marcos, A., Balas, G.J., Bokor, J.. Linear paramater-varying detection filter design for a boeing 747-100/200 aircraft. Journal of guidance, control, and dynamics 2005;28(3):461-470. 


Nomenclature
TP Two Phase
SH Superheated
SC Subcooled
N Compressor Speed
T Temperature
a air
c Condenser
cmp Compressor
e Evaporator
g gas
h Enthalpy
l liquid
p Pressure
v Valve
$\dot{m}$ Mass Flow Rate
$\dot{Q}$ Heat Transfer Rate
$\alpha$ Valve Position
$\gamma$ Void Fraction
$\delta$ uncertainty
$\rho$ Density
$\zeta$ Normalized Phase Region Length
$\mu$ Structured Singular Value

